\title{
Acoustic System for Online Wiring Test on Aircraft
}

\author{
Z. Xu ${ }^{1}$, D. Koltsov ${ }^{1}$, S. Saha ${ }^{1}$, A. Richardson ${ }^{1}$, A. Sutherland ${ }^{2}$ \\ ${ }^{1}$ Centre for Microsystems Engineering, Engineering Department, Lancaster University, Lancaster, LA1 4YR, UK \\ ${ }^{2}$ Ultra Electronics - BCF, Phoenix House, Phoenix Way, Cirencester, GL7 1QG, UK
}

\begin{abstract}
Wiring onboard of legacy aircraft is becoming a liability due to a prolonged use of aircraft. Testing as well as repairs and maintenance require extensive number of man-hours bringing up the costs and reducing flight time of the aircraft. There is a number of conventional electric testing methods for wire testing, yet we propose and evaluate an acoustic (mechanical) testing method. Acoustic testing can be used online while the wire is in operation, and has the capability for intermittent fault detection.We report modelling and experimental results of an insulated wire that indicate this technique can be used to detect mechanical faults in insulation and conducting wire core. We used two different methods to detect and locate insulation defects of only $0.2 \mathrm{~mm}$ wide. Transmission mode allowed a clear detection of the defect while reflection mode yielded the defect position.
\end{abstract}

\section{INTRODUCTION}

Recent developments in aviation manufacturer and avionics equipment maintenance communities have highlighted a major oversight in electrical wire testing. This applies to the maintenance of aging aircraft $[1,2]$ as well as the design of novel types of aircraft. In addition, we have recently witnessed a drive for a more reliable and safe air travel. In normal maintenance regime of an aircraft safety costs a lot of maintenance man-hours and in the case of wiring testing this becomes a very costly procedure. In US military aviation alone the wire testing and maintenance takes 1 million man-hours per year with a bill of \$10M for replacements [3]. Oversight during this maintenance led to aborting of 1400 missions per year.

The problem is therefore real and requires a systematic approach. According to the survey for US military aircraft, just connectors and conductors alone contributed to $14 \%$ and $29 \%$ of incidents respectively. When the faults were identified, it turned out that chaffed wire insulation, wire short circuits and broken wires contributed to $37 \%, 18 \%$ and $11 \%$ of incidents respectively. Even though relatively few of wiring faults lead to disasters, they contribute to the down time of aircraft.

Surprisingly, even to date most of the wiring inspections are performed visually. This is not only a laborious task but is also not a very reliable one. There is a large selection of more reliable testing methods such as reflectometry (frequency and time-domain) [4] or resistance or peak voltage tests. But most of these advanced techniques lack the ability to detect mechanical (not electrical) faults. Such mechanical faults usually lead to electrical failure thus making these techniques preventative. Another problem is to detect and identify intermittent faults that are undetectable when the aircraft is not in use.

We propose to use acoustic (mechanical) means of testing the integrity of structures and will concentrate on our simulation and experimental results. This paper aims to take the original ideas by Anastasi [5] further in order to quantify the merits of the acoustic wire testing [6]. This work extends the range of acoustic signal propagation as well as introduces a time-domain detection of a crack in a wire system. We describe our modelling results and show that a detectable signal for a simplified metal rod with insulation works very well. Modelling section is followed by the experimental results where we demonstrate a clear detectable signal from a defect in insulation.

\section{PROPOSED METHODS}

The advantage of acoustic testing method is that it uses mechanical stimuli instead of the traditional electrical signals, thus it could be applied online while the wires are operating, which means that finding intermittent faults becomes possible. It also provides the capability of monitoring the insulator layer as well as the conductors.

We propose to send an acoustic signal through the wire. The mechanical imperfections, defects and faults are to be detected though measurements of transmitted and reflected signals. Figure 1 shows the proposed structure. The key components are pairs of piezoelectric acoustic transducers. These transducers can produce transverse and/or longitudinal waves along the rod, and are fixed onto the wire at certain distance away from each other. Each of the transducers can be configured as acoustic emitter or receiver independently. Two types of transducers have been proposed: the connector type which can be integrated into the conventional connectors, and the clip type which could be just clamped onto a wire. The connector type has advantage of injecting acoustic testing signal directly into the conductor of the wire. It is much more efficient on transmitting signals compared with the clip type, as the insulator layer of the wire is a poor acoustic conductor. While on the other hand, the clip type is more sensitive to the defects which exist only in wire insulation.

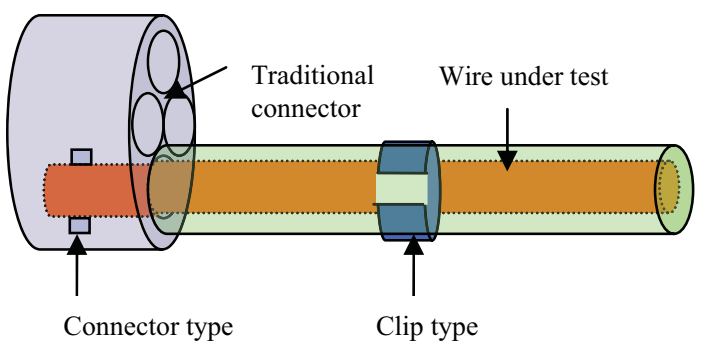

Fig. 1. Two types of proposed transducers. 
Defect detection can be achieved by locking at spectral signatures of the transmitted signal or by simply comparing the signal to a pre-stored signal for the defect-free system. There are two modes of test: the pulse-echo mode and the through mode. In the pulse-echo mode, the same transducer is used as the emitter and the receiver. It firstly sends out a burst of testing signals, and then records the reflection. In the through mode, one transducer on the wire sends out a test signal, the other transducer next to it is configured as receiver.

\subsection{MODELLING OF THE WIRE}

Finite element analysis has been performed to evaluate the feasibility of acoustic wire testing method. A model of the MIL-W-81381/7 Gauge 20 has been built as shown in figure 2 . The wire has polyimide insulator (internal diameter $=0.942 \mathrm{~mm}$, outside diameter $=1.286 \mathrm{~mm}$ ). As shown by figure 2, a stress wave has been applied on one end of the conductor as the test input. The stress wave is injected at the input end into the conductor. The acoustic signal travels through the structure, but the output is detected from the surface of insulation layer and from the conductor that was originally stimulated. A small crack in the insulator layer is also added in the middle of the wire. The width of the crack is $0.2 \mathrm{~mm}$ and the depth changes from 0 to the full thickness of the insulator.

The attenuation of an acoustic wave is normally proportional to the square of its carrier frequency, thus the frequency of the input test signal $(f)$ needs to be chosen carefully. If $f$ is too low, the wavelength of the stress wave will be big and non comparable to the crack size, thus no defect could be detected. However, if $f$ is too high, the attenuation of the signal will be strong, especially in flexible material like the insulator. In this case frequency of $1 \mathrm{MHz}$ is used.

\subsection{Simulation of Pulse-Echo Mode}

In this measurement a test signal of a burst is applied at the conductor input end (figure 2), and the reflection is monitor at the same position. The burst is a shifted and scaled Morlet wave, defined by equation 1 .

$$
A= \begin{cases}A_{0} e^{-x^{2} / 2} \cos (5 x) & \text { When } 0<t \leq 6.4 \times 10^{-5} \\ 0 & \text { When } t>6.4 \times 10^{-5}\end{cases}
$$

where

$$
x=t \times 1.25 \times 10^{5}-4
$$

In equation $1 A$ is the amplitude of the input signal, $A_{0}$ is the magnitude, $e$ is the Napier's constant and $t$ is the time. Figure 3 shows a burst of the normalized input signal.

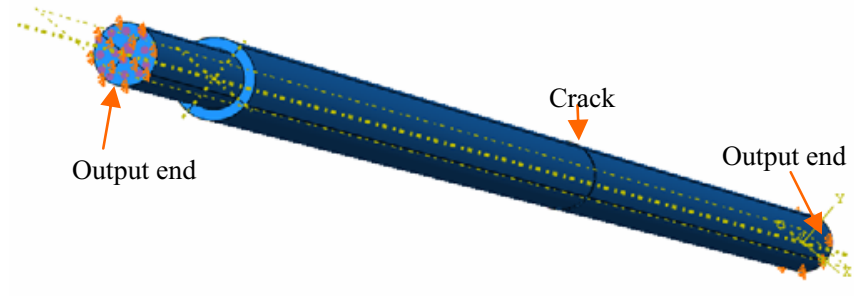

Fig. 2. Model of a wire with crack in insulator layer.

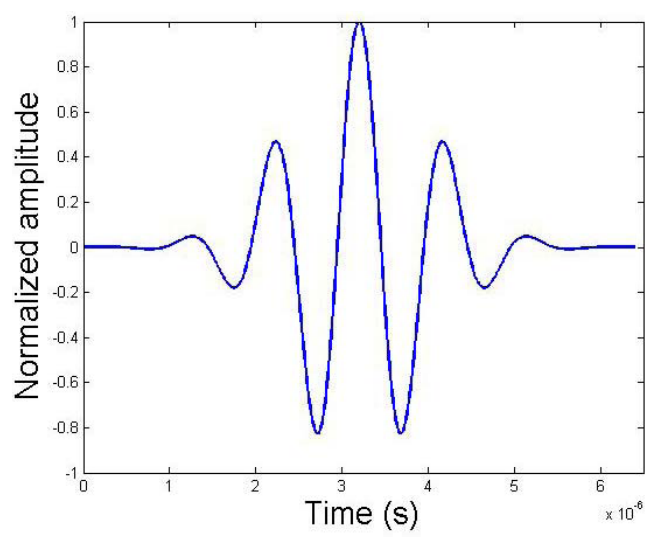

Fig. 3. Normalized burst input.

Fully cut crack in the insulator at 2 different positions are simulated. The reflections received from the defect wire are compared to the recorded reflection of a healthy wire. Figure 4 shows the differences of the displacement output between defect and healthy wires. It is clear that the arriving time of the differences indicates the location of the crack.

\subsection{Simulation of Through Mode}

In the simulation of through mode, the location of the crack is fixed in the middle of the wire. The depth changes from 1/6 to the full thickness of the insulator layer. The input testing signal in this case is a continues sine wave at frequency of $1 \mathrm{MHz}$. The displacement and stress at the insulator output end is monitored and compared to the healthy state. Figure 5 shows the Fast Fourier Transfer (FFT) result of the displacement output, with a crack of $0.2 \mathrm{~mm}$ wide and half thickness cut of the insulator layer. It is clear that the defect can be indicated by the major peak at $1 \mathrm{MHz}$. Figure 5 indicates that the carrier frequency has higher transmission in the case of healthy wire. Cracked wire however seemed to enhance the transmission of frequency side bands.

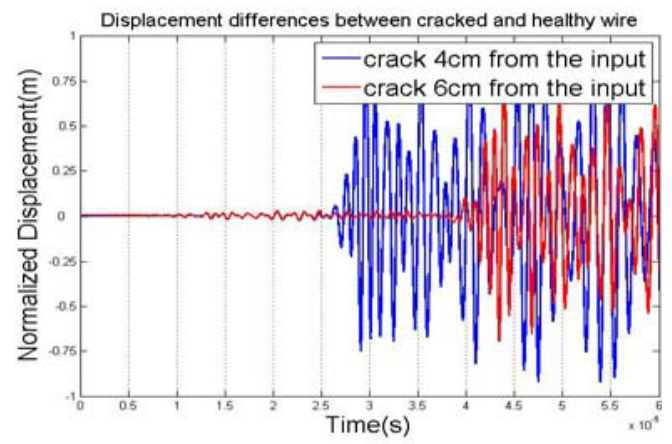

Fig. 4. Time-domain detection of echo mode with insulation crack at $4 \mathrm{~cm}$ (blue) and $6 \mathrm{~cm}$ (red). 


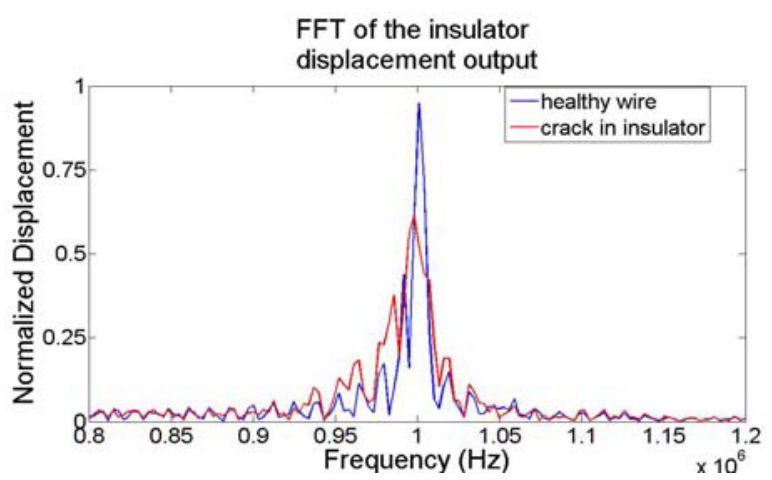

Fig. 5. Transmitted signal FFT for healthy and cracked insulation wires

\section{EXPERIMENTAL SETUP AND MEASUREMENTS OF THROUGH MODE}

A through mode experimental setup is constructed to compare the simulation result described in section 2.3. In the experiment, we have used off the shelf components (transducer) and test structures (steel rod and heat shrink coat) as an initial setup. Instead of real wire, we have used steel rod as the core of the wire and heat shrink insulation as the cladding of the wire.

The attenuation of the acoustic wave is very high in insulation and increases significantly with frequency for both steel and insulation. So a relatively low frequency $(500 \mathrm{KHz})$ piezoelectric transducers (Panametrics NDT V151 $0.5 \mathrm{MHz}$ ) were chosen for the experiment. In the measurement setup, one transducer works as an emitter and a second transducer works as a receiver as shown in Figure 6. Each transducer can work as both emitter and receiver. The emitting transducer is placed directly in contact with the steel rod and the receiving transducer is placed on the insulation. Both transducers are identical and operate in transverse wave propagation mode. The distance between the transducers is $14 \mathrm{~cm}$ and the diameter of the steel rod used as core is $6 \mathrm{~mm}$. The thickness of the heat shrink insulation (polyolefin) used as cladding is 0.5 $\mathrm{mm}$.

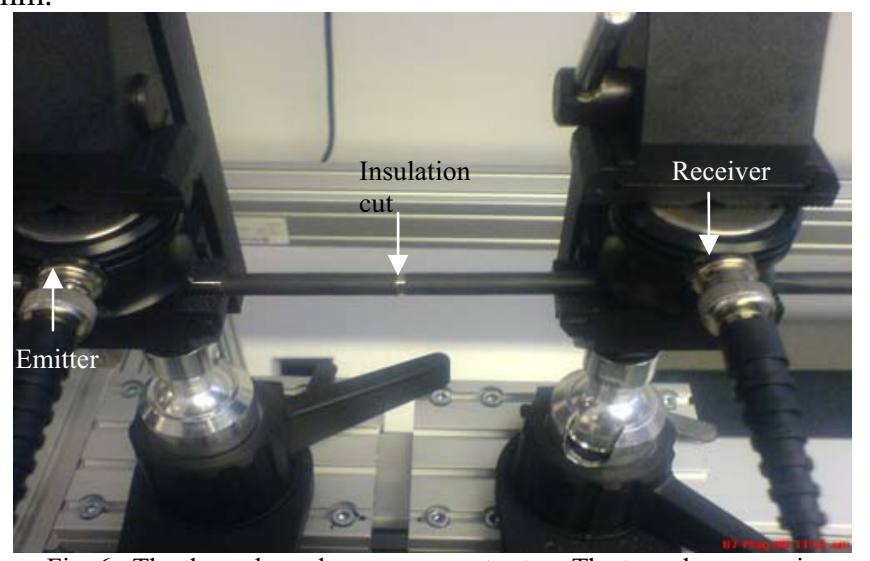

Fig. 6. The through mode measurement setup. The transducers are in transverse mode and $14 \mathrm{~cm}$ apart. The emitting transducer is on the steel and the receiving transducer is on the insulation. A full cut is introduced in the insulation.

\section{Frequency response in transverse mode}

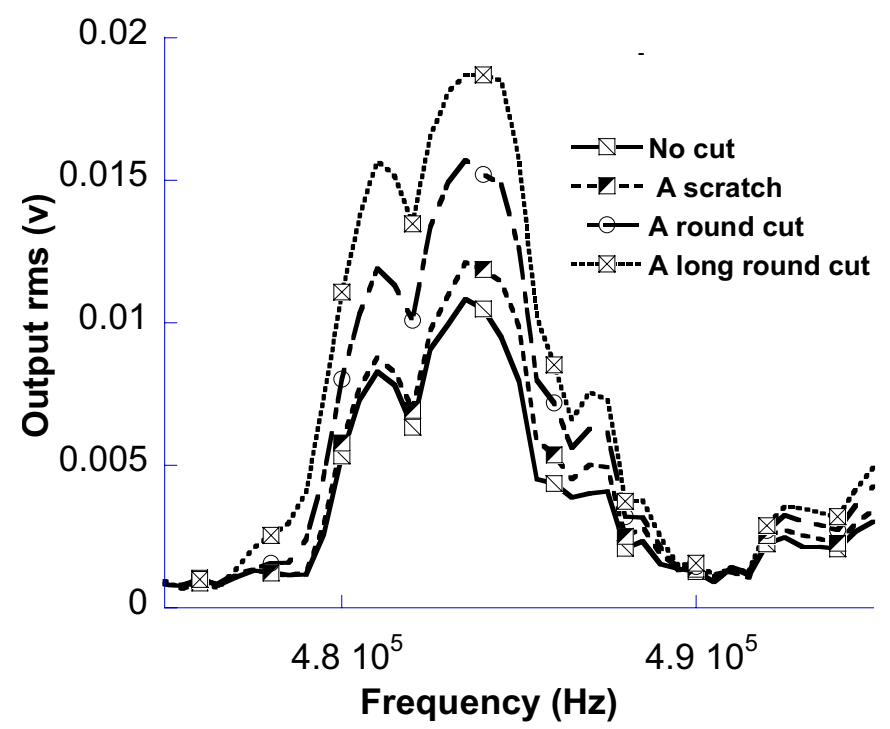

Fig. 7. The rms value of the received signal with various type of cut on the insulation. The transmit transducer is on steel and receive transducer is on insulation.

A $1 \mathrm{~V}$ peak-to-peak alternating current (AC) signal is applied to the emitting transducer. The frequency of the emitting signal is varied from $450 \mathrm{KHz}$ to $600 \mathrm{KHz}$. The acoustic transverse wave propagates through the steel rod and insulation. The receiving transducer, placed on insulation, detects mechanical vibration. A Tektronics AFG3022 dual channel arbitrary function signal generator is used to generate the test signal. An HP54645A $200 \mathrm{MSa} / \mathrm{s}$ oscilloscope is used to monitor the received signal. Both signal generator and oscilloscope are controlled by a PC running Labview program and interface. The root mean square (rms) value of the signal obtained from receiving transducer is measured and compared for various types of insulation cuts. The comparison is shown by Figure 7. In Figure 7, No cut means, the insulation is in good condition, A scratch means, a scratch is introduced in the insulation by knife, A Round cut means, a $2 \mathrm{~mm}$ wide cut is introduced in the insulation around the rod (shown in Fig. 6) and $A$ Long round cut means, a $1 \mathrm{~cm}$ wide cut is introduced in the insulation around the rod.

From the measurement results, it can be seen that the signal transmission increases with the increase in the amount of cut in the insulation. The transverse wave transmission is very sensitive to the insulation and absorbed significantly during propagation through the rod. So by comparing the received signal strength of a test wire with a healthy wire, we can detect a cut or crack in the wire.

\section{CONCLUSION}

To summarize, we have proposed an acoustic online wire testing system. A series of experiments and simulations have been performed to access the feasibility of the above system. Both experiment and theory results indicate high sensitivity of 
the system to a fault in wire insulation. The experiment results show that a system with larger insulation break transmits acoustic signals better than the healthy wire. This wire testing method is therefore applicable to more complex systems onboard of aircraft, due to its sensitivity and relative simplicity.

\section{References}

1. Johnson, D.H.W., Edward L. ; D'Angelo, Joseph J., Jr. ; Dicks, Dwayne ; Decker, Adam L., Wiring System Diagnostic Techniques for Legacy Aircraft. Conference Publication, 2003.

2. Furse, C.H., R; , Down to the wire. IEEE Spectrum, 2001. 38(2): p. 34-39.

3. Sunderland, A., Health Monitoring for Aerospace Wiring Systems, in HUMS EU consultation meeting at Lancaster University (UK). 2008: Lancaster.

4. Furse, C.Y., C.C. ; Dangol, R. ;Nielsen, M.; Mabey, G. ; Woodward, R. , Frequency-domain reflectometry for on-board testing of aging aircraft wiring. IEEE Transactions on Electromagnetic Compatibility, 2003. 45(2): p. 306- 315.

5. Anastasi, R.F.M., E.I. ; , Application of ultrasonic guided waves for aging wire insulation assessment. Materials evaluation, 2005. 63(2): p. 143-147.

6. Zhao, X.Q., T. ; Popovic, Z.; Zane,R.; Mei, G. ;Walsh,C. ;Paing, T. ; Kwan, C. . A Wireless Ultrasonic Guided Wave Structural Health Monitoring System for Aircraft Wing Inspection. 2007: AIP Conf. Proc.

7. C. Teal, C.S., Managed aircraft wiring health directly relates to improved avionics performance. 19th Digital Avionics Systems Conferences, proceedings, 2003. 